\title{
Lecture \\ The Basis in Ontogeny of Language Ability
}

\author{
HARUMI KOBAYASHI \\ Tokyo Denki University
}

\begin{abstract}
Social ability seems to play a major role in the ontogeny of language. Human babies are already sensitive to adults' eye gaze, and follow it at an early age. At the first year of life, they begin to redirect the adults' attention and actively establish joint attention with caregivers and other important adults. They then use the established joint attention to guess meanings of words they hear. Later, children further show socio-pragmatic ability at a higher level to guess word meanings. They still use joint attention but they may not depend upon joint attention in some situations. Flow-of-conversation and guessing others' internal conditions can also give important cues for language development.
\end{abstract}

There are many approaches to explore the origins of language, such as those in guessing use of language based on fossil evidence on brain size and speech organs, studying the communication of non-human primates or other animals, and constructive approaches including computer simulations. Compared with these approaches, studying how human infants acquire language seems to have a special advantage in the examination of the origins of language. The reason is that the ontogeny of language ability can be observed in real time in front of the eyes of researchers, so in theory, we can observe every change from a micro level to a macro level. Human infants do not have language at birth, but later acquire it. The human species did not have language when their ancestors appeared on the earth but later acquired it. Of course, there is a huge difference in the time scales of these two developments. In the former case, the development takes around 5 years or more depending upon specific abilities of language, in the latter, around 200,000 years or more depending upon the concept of the basis of language ability. However, it seems to be a

Published online in J-STAGE: June 18, 2010 doi: 10.2502 janip.60.1.6 fruitful assumption that at least some mechanism to propel these two language developments is shared. Thus, the ontogeny of language ability may be studied in parallel with the phylogeny of language.

\section{Development of Visual Joint Attention}

Joint attention, or one child and one adult paying attention to the same object in the environment, is estimated to be one of the most important forces to promote early language acquisition in developmental psychology. Joint attention can be established on any kind of sensory stimulus, but visual joint attention is supposed to be a major factor for typically developing children. In their classic and still influential work, Scaife and Bruner (1975) first showed that babies have the ability to achieve visual joint attention. They asked a mother to put her baby on her lap and an experimenter first established eye-contact with the baby, and then the experimenter looked in either a left or right direction. The baby's direction of eye gaze was recorded. The babies were 2- to 14- month-olds. The results were that some 2-month-olds looked in the direction the experimenter looked. At 14 months, $100 \%$ of babies did so. Butterworth and Jarrett (1991) adapted Scaife and Bruner's paradigm and 
found that at 6 months, babies looked in the same general direction. At 12 months, babies looked at a specific stimulus in their visual field. At 18 months, babies looked back to look at a specific stimulus behind their visual field.

\section{Nine-Month Revolution}

Tomasello (2001) claimed that at around nine-months, special changes that are distinctively different from other primates are observed. Before nine months, a child either attends to an object such as playing with a toy train, or to a person such as enjoying pee-ka-boo with his mother. The interaction is basically dyadic and the child does not attempt to attend to both the object and the person of his interest simultaneously. However, the interaction pattern changes after ninemonths. The child now attends to both the object and the person of his interest simultaneously. Children typically start a series of actions that demonstrate their newly developed ability of interaction. They check their mothers' eyes to know whether their mothers properly look at and pay attention to the same object they look at, and if their mothers do not look at it, they point at it uttering a word or an attention-getting vocalization. Or they hold the object, bring it to their mothers and show or give it to them. Here, the major interaction pattern is triadic, that is, interaction between child, object, and person. Tomasello named this change the "Nine-Month Revolution" to emphasize the emergence of this fundamental aspect of human communication.

\section{Five Stages of Joint Attention}

After reviewing previous discussions and experimental results, Ohyabu (2004) claimed that joint attention develops through five different stages: Pre-joint Attention (appears after birth to 2 months), Face-to-face Joint Attention (2 mo. - 6 mo.), Supported Joint Attention (6 mo. - 9 mo.) Intention Sharing Joint Attention (9 mo. - 12 mo.), and finally, Symbol Sharing Joint Attention (15 mo. - 18 mo.). Tomasello's nine-month revolution can be placed at the Intention Sharing Joint Attention phase. Pre-joint Attention refers to newborn and very young babies' inclination toward human faces, eyes, and voices. Adults are also sensitive to babies' signals mediated by babies' faces, bodily actions, and voices. Pre-joint attention appears in an innately specified, primitive, and weak interaction between babies and caregivers. At Face-toface Joint Attention, the baby is obviously looking at the caregiver's eyes, and the baby shows socially originated smiles. Around 6 months or later, Supported Joint Attention appears. It can be established in two ways, a caregiver may detect the direction of the infant's eye gaze and turn to gaze at the object, or an infant may detect the direction of the caregiver's eye gaze and turn to gaze at the object. Ohyabu pointed out that at this stage, the caregiver's role seems more important for establishing joint attention. The frequency of the caregiver turning her eyes toward the object is higher than the infant turning his eyes toward the object.

The infant's relatively passive role dramatically changes at around nine-months and later. Intention Sharing Joint Attention appears at this stage and it refers to an infant's active behavior of detecting and controlling the direction of an adult's eye gaze. The infant checks the adult's direction of eye gaze and if the person does not look at the object, the child starts to perform a variety of attention sharing actions such as pointing, showing, and giving. The child also starts to think recursively. She starts to recognize that she is seen by the adult and later makes use of this knowledge to understand others' intentions. At the Symbol Sharing Joint Attention that appears between 15 and 18 months, language produced at the presentation of an object can be associated to the object. The child can share the meaning of the other's intentions and consequently can learn the meanings of symbols (words) that are uttered in the joint attention situation. Later the child will be able to establish joint attention about the objects that are not present, because the 
object of joint attention can be both concrete and abstract. The child will start to use symbols to refer to absent referents, such as past and future events. Thus language acquisition is made possible.

\section{Joint Attention and Early Language Develop- ment}

Is joint attention really important in children's word learning? The role of joint attention in early vocabulary development was nicely demonstrated by Tomasello and Farrar (1986). In their observational study (Study 1), 24 children were video-taped at 15 and 21 months of age in naturalistic interaction with their mothers. Joint attention episodes such as joint play with an object were identified, and in these episodes 'Inside' joint attention situations and 'Outside' joint attention situations were further identified. Inside Joint Attention refers to the situation that the mother follows the child's focus of attention, that is, the child's attention is already inside of joint attention, and the mother provides a name for it. Outside Joint Attention refers to the situation that the mother directs the child's focus of attention to an object that the child does not attend to, that is, the child's attention was initially outside of joint attention, and the mother provides the name for it. In Inside as opposed to Outside Joint Attention, mothers and children produced more utterances, mothers used shorter sentences and more comments, and dyads engaged in longer conversations. Maternal references to objects that were already the child's focus of attention were positively correlated with the child's vocabulary at 21 months. However, object references that attempted to redirect the child's attention were negatively correlated. In Tomasello and Farrar's experimental study (Study 2), an adult attempted to teach novel words to ten 17 month-old children. They found that the words referring to objects on which the child's attention was already focused were learned better than the words presented in an attempt to redirect the child's attentional focus.

\section{Not Depending upon Joint Attention}

However, joint attention may not be necessary in some situations. Tomasello and Barton (1994) showed that even when ostensive definition was not presented, i.e., even when a label was presented but the referent of it was not presented simultaneously, two-year-olds were still able to know word meanings. In their experiment, the experimenter first announced that she would find "a toma." Then she opened the first two buckets out of four buckets, looked inside of each, took out an object from each bucket and showed disappointment. But when she opened the third bucket, she took out an object and gave it to the child showing excitement and joy. At this time she did these things without labeling the objects at all. Later the child was asked to choose "toma" out of five objects. Two-year-olds had little difficulty in choosing the correct object. Here joint attention was not necessary. The child knew what the experimenter wanted at the beginning of the experiment, and held that knowledge throughout the experiment. The child also had knowledge about the relationship between facial expression and emotion, and used the knowledge to find whether the experimenter found what she wanted, and was able to successfully specify the object named "toma."

In the Tomasello and Haberl (2003) study, a child use the knowledge that other people know the newly added toy or not to guess the name of a novel toy. First, the child and two experimenters played with two novel toys. After a while, Experimenter A left the room and the child continuously played with Experimenter B. Experimenter B added another novel toy and the child and Experimenter B played with the three toys. Then Experimenter A came back to the room, looked at the playing situation and said to the child "Woo! Give it to me!" The child correctly gave the newly added toy. When a novel label was used instead of "it," the child again correctly chose the newly added toy to the person who left the room. Here, again ostensive definition was never 
given. The child's ability to guess the internal condition of the adult who left the room and later returned was important to know the referent. These studies clearly demonstrate that children's social pragmatic ability plays an important role to know the referent of the adults' words. Beyond ostensive definition and beyond the firsthand joint attention situation, children can acquire word meanings. It is said that more than half of adults' labeling in everyday situations is not ostensive definition (Tomasello, 2001). Considering the nature of everyday situations where ostensive definition is not always given, this ability to guess others' internal condition must be very useful in learning words.

\section{Use of Social Cues in Word Learning}

More recent studies show details of young children's social ability in word learning. Infants properly follow adults' gaze direction (Brooks \& Meltzoff, 2002; Moll \& Tomasello, 2004). Infants also have an ability to actively consult cues that speakers provide to know the reference of their utterances (Golinkoff \& Hirsh-Pasek, 1999; Hirsh-Pasek, Golinkoff, \& Hollich, 2000). Among different cues for word learning, cues of adults' social intent seem to be well established. For example, Hollich, Hirsh-Pasek, and Golinkoff (2000) examined the effects of competing perceptual and social cues on word learning in 12-, 19-, and 24-month-olds. Children were presented with novel objects, one interesting and one boring, and given the opportunity to explore each one. Then the objects were placed on the board and the experimenter enthusiastically looked at, pointed to, and labeled either the interesting (coincident) or boring (conflict) object. Children were later tested to choose the referent of the novel object. The objectlooking time of each child in each condition was examined. The results were that 19- and 24-month-olds looked at and chose the socially intended object as a referent even when the object was boring, but 12-montholds tended to look at and choose the interesting object as a referent regardless of the existence of the social cue. Hollich et al. interpreted this result that children make a shift in word learning from relying on perceptual cues to relying on social intent cues.

\section{Discrepant Labeling Situation}

In a discrepant labeling situation that Baldwin (1991, 1993) examined, infants would establish an incorrect word-object mapping if they failed to recognize the significance of cues such as line-of-regard for determining the speaker's reference. In this paradigm, an experimenter showed infants two equally attractive novel toys and gave one to the child to play with while the experimenter retained the other. When the infants' attention was focused on the toy, the experimenter looked at and labeled her own toy, the situation being a discrepancy between the infants' focus and that of the speaker at the time the label was uttered. Without the ability to consult the speaker for cues to reference, infants should fall prey to a mapping error because of the time contingency that would lead them to link the new label with the toy of their own focus. On the other hand, if infants appreciated the relevance of the speaker's non-verbal cues such as line-of-regard and body orientation directed toward the speaker's toy, they could use these cues and establish the correct word-to-object link. According to Baldwin, infants readily glanced up at the experimenter when discrepant labeling occurred and followed her gaze to the object of her focus. In a comprehension test, infants of 18 months or older did not link the new label with the toy they were actually focused on when they heard the new label. Eighteen- to nineteen-month-olds seems to have established a stable mapping between the label and the object of the speaker's focus. These results suggest that infants have the ability to appreciate that speakers provide cues that are relevant to interpreting new words and consult these cues when faced with discrepant labeling. Baldwin's studies successfully showed infants' sensitivity to know the 
others' direction of attention by confirming the nonverbal cues of eye gaze and face or body direction of others, but children also seem to have abilities to use relevant cues that are beyond such physical cues of eye, face, and body.

\section{An Empirical Study of Pragmatic Cues}

In our previous study, Kobayashi and Yasuda (2007) tested two-, four-year-olds and adults in this discrepant labeling paradigm and were surprised with unexpected results. We expected that most 2-year-olds would readily check the experimenter's face in the experiment of the discrepant labeling paradigm. However, despite our expectation, nearly 40 percent of the 2-year-olds who had previously looked at their chosen object at their hand (In-Attention-Object) immediately changed their focus of attention and took the other object that they did not choose (Out-ofAttention Object) WITHOUT looking at the experimenter. Among the 4-year-olds, this tendency was more distinct. About half of the children who previously looked at their chosen object similarly changed their focus of attention and took the other object that they did not choose without looking at the experimenter. We tested adults in virtually the same paradigm and found that about 70 percent of the adults similarly changed their focus of attention when they heard a label without checking the experimenter's face or body direction. Why did these children and adults not seem to check the direction of the experimenter's eye gaze? We speculated that they were likely aware that the experimenter may not necessarily say the name of the object the participant was attending to. Even young children seemed to think that the label must be the referent of the other object which is not the present focus of the child. If they heard the experimenter label something after they had already played with the object for 10 seconds, they seemed to think that the object they had at hand was not the object the adult labeled. However, if the children heard the experimenter immediately label something after they took it, they seemed to think that they needed to check the adult's line-of-regard to know what was labeled. If this is the case, young children certainly rely on social pragmatic cues in a sense, with a more sophisticated way than simply checking the adults' line-of-regard.

A stronger test of this hypothesis may be to contrast children's responses in two alternative situations: modifying the amount of time elapsing between when the child began playing with one object, and when the experimenter labeled the other novel object. Thus, in one situation, the child hears the novel word immediately after she touches the object she chose, and in another situation, the child hears the novel word after 10 seconds elapsing after her touching the object. If children look at the Out-ofAttention Object immediately after touching the chosen object only in the 10 secondelapsing condition, the social pragmatic explanation can be more strongly validated. In our study (Yasuda \& Kobayashi, 2008) we tested our hypothesis of social pragmatic cues in this more stringent test.

Participants were thirteen 2-year-olds (range 2;0 3;4) and 15 4-year-olds $(4 ; 1 \sim 5 ; 0)$. Figure 1 shows the experimental situation. The child and the experimenter sat on a table face-to-face. On the table, the experimenter placed the two novel objects in front of the child. The two objects were at an equal distance from the child (Scene 1). The experimenter said to the child, "Which do you like? You can play with the one you like." Then the child took one of the objects and manipulated it (Scene 2). When it was confirmed that the child was focused on the chosen object, the experimenter immediately looked at the other object that the child did not choose and said, "Oh, (child's name), muta, muta!" (Scene 3). The child's response was noted. Immediately after each trial of labeling, the experimenter presented the two previously shown objects and asked the child, "Which is muta?" The child's responses, both verbal and nonverbal were noted.

The object the child looked at was coded into four categories; Experimenter, In- 


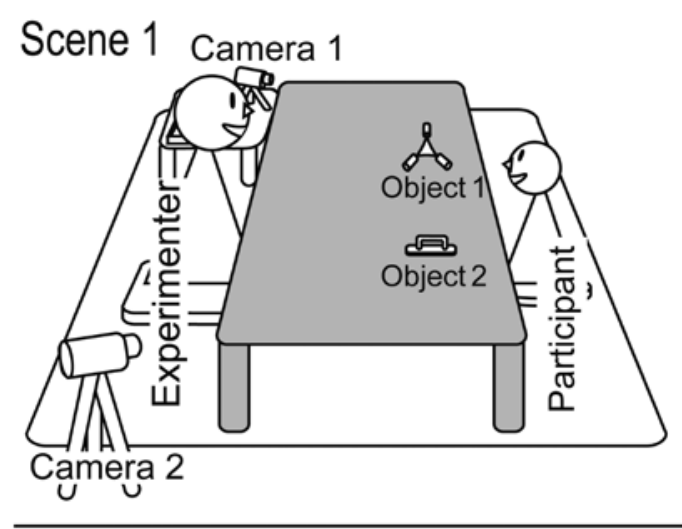

\section{Scene 2}
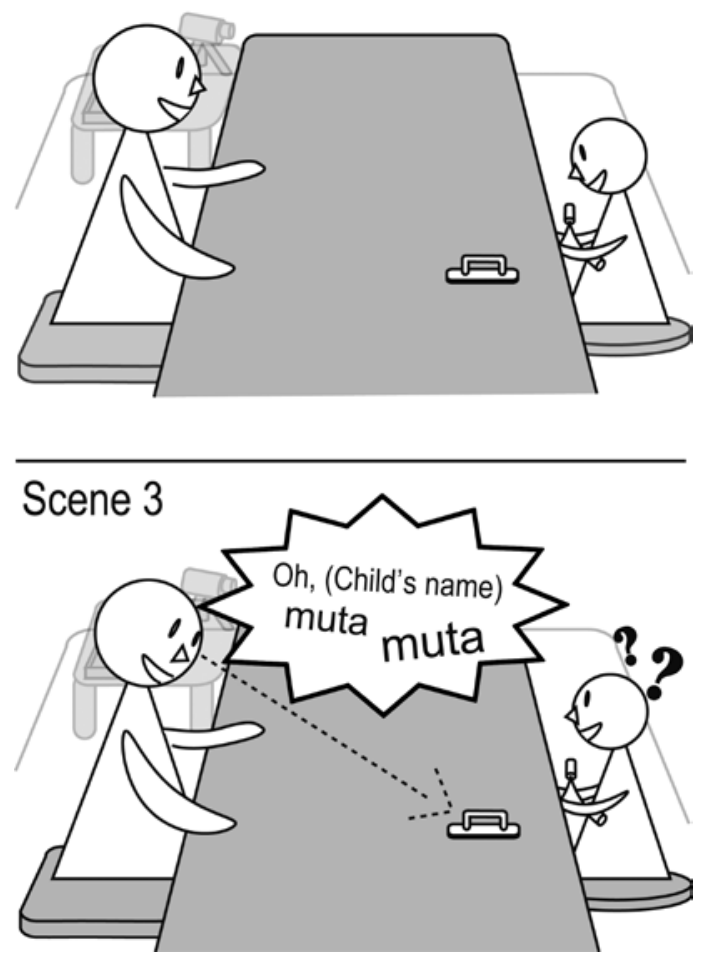

Figure 1. The Experimental Situation of Discrepant Labeling

Attention Object, Out-of-Attention Object, and Other, for example, the child's line-of-regard was coded Experimenter when the child looked at the experimenter's face or body. The mean ratio of change of line-of-regard from one category to the other category was calculated for each movement.

Figure 2 shows the mean frequency of the line-of-regard to the experimenter immediately after or after 10 seconds elapsed. Statistical analysis revealed that children of both ages checked the experimenter's face more in the immediate (0 second) condition than in the 10 second condition.

The result of eye gaze analysis was that in the 10 second condition, a substantial ratio of the 2 -year-olds (37\%) looked at the Out-of-Attention Object without checking the experimenter's face. The ratio of this direct looking of Out-of-Attention Object was 33\% in the 0 second condition. Among 4-year-olds. $46 \%$ of them looked at the Out-of-Attention Object without checking the experimenter's face in the 10 second condition whereas only $27 \%$ of them did so in the 0 second condition. These results suggest that children become able to look at an appropriate object without checking others' eye gaze at least by age four.

We think the most interesting result of this study was the fact that the children did check the experimenter's eye gaze when the experimenter looked at and labeled the Outof-Attention Object immediately after the child took the object of her choice. When we compare the data of the 0 second condition and the data of the 10 second condition, we can say that young children do not always check the direction of the line-of-regard when they hear a novel word, if they have a good reason to associate the given label to a certain object. If they hear the experimenter label something after they had already played with the object for 10 seconds, they seem to think that the object they have at hand is not the object the adult labeled. However, if the children hear the experimenter immediately label something after they took the object, they seem to think that they need to check the adult's line-of-regard to know what was labeled. Children may think as follows. If the speaker wanted to comment on the object that the child chose and manipulated, she should have commented earlier. Because she commented after 10 seconds had passed since the child had started to manipulate the object, she must have intended to make an inference about 


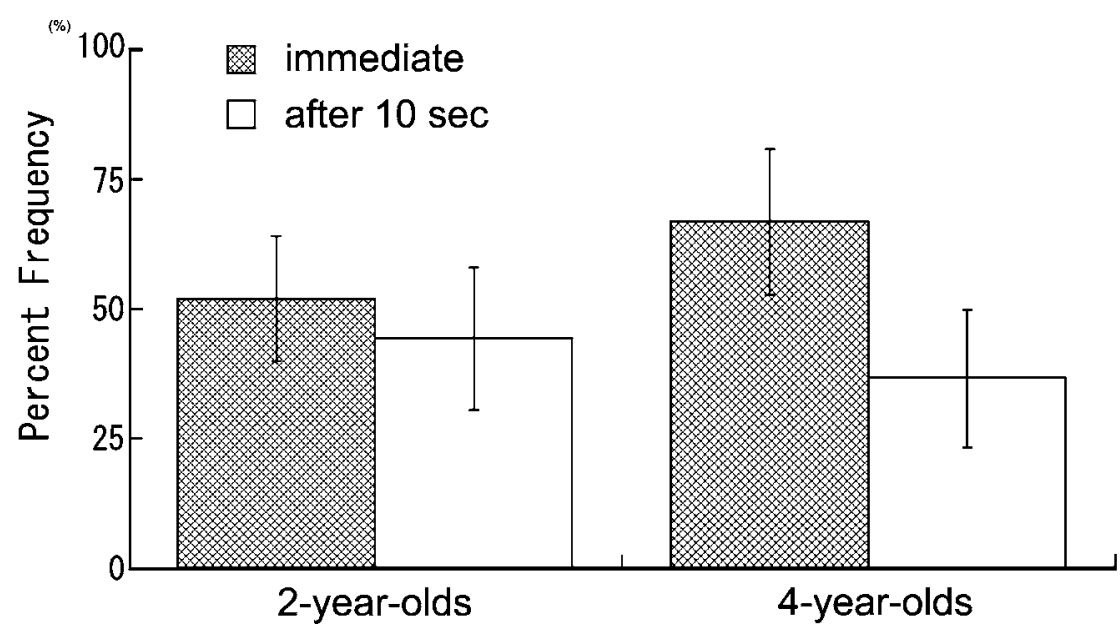

Figure 2. The mean frequency of the line-of-regard to the experimenter immediately after or after 10 seconds elapsed

the object which had not been the already chosen.

\section{Social Intent as a Cue for Guessing Word Meanings}

The result of this study supports the recent contention of the importance of the flow of conversation proposed by Tomasello and colleagues (Tomasello \& Akhtar, 1995; Tomasello \& Haberl, 2003). In several studies Tomasello and Akhtar demonstrated that young children have the ability to know the referential intentions of adults even when seeing an object and hearing the label of it do not occur simultaneously. One of the most important things for children to know in learning object names seems to know the adult's desire and hopes and how such internal information may appear in facial expressions and tone of voice. We think our study has added a new insight to this concept of the flow of conversation by examining the effect of immediate labeling and comparing immediate labeling and labeling after 10 seconds elapsed. Even the consecutive manipulation of only 10 seconds may be enough for children to conclude that the already manipulated object must not be the object that the adult commented on with a nuance of excitement. The result of this study also partly supports the recent contention of a social shift in word learning proposed by Golinkoff, Hirsh-Pasek, and colleagues (Golinkoff \& Hirsh-Pasek, 1999; Hirsh-Pasek, Golinkoff, \& Hollich, 2000). They state that children come to rely on social intent as a more reliable cue than other cues such as perceptual saliency in word learning after around 18 months of age. This study provides evidence that children may further develop using two different types of social cues depending upon the situation, i.e., lineof-regard of other people (a perceptual social cue) and timing of utterances (a pragmatic social cue). As children get older, this kind of sensitivity to social cues that reflect adult intent are sharpened and used for further word learning. In a more recent study, Yasuda and Kobayashi (2010) showed that this ability develops between ages 2 and 3 . They suggested that 3 -year-olds are already sensitive to timing of adults' utterances and depended upon the timing information more than adults' eye gaze information to guess word meanings using a similar discrepant labeling paradigm.

\section{Conclusion: Studying Ontogeny of Language} Ability from a Socio-Pragmatic Perspective

The reviewed studies suggest that social ability seems to play an important role in the ontogeny of language. Human infants are 
already sensitive to adults' eye gaze, and follow it at an early age. At the first year of life, they begin to redirect the adults' attention and actively establish joint attention with caregivers and other important adults. They then use the established joint attention to guess meanings of words they hear. Later, children further show socio-pragmatic ability at a higher level to guess word meanings. They still use joint attention but they may not depend upon joint attention in some situations. Flow-of-conversation and guessing others' internal conditions can also give important cues for language development.

\section{Acknowledgements}

This study was partly supported by KAKENHI (20500241) and KAKENHI (20246071).

\section{References}

Baldwin, D. 1991. Infants' contribution to the achievement of joint reference. Child Development, 62, 875-890.

Baldwin, D. 1993. Early referential understanding: Infants' ability to recognize referential acts for what they are. Developmental Psychology, 29, 832-843.

Bowerman, M., \& Levinson, S. (Eds.). 2001. Language Acquisition and Conceptual Development. Cambridge University Press.

Brooks, R., \& Meltzoff, A. N. 2002. The importance of eyes: How infants interpret adult looking behavior. Developmental Psychology, 38, 958-966.

Butterworth, G., \& Jarrett, N. 1991. What minds have in common is space: spatial mechanisms serving joint visual attention in infancy. British Journal of Developmental Psychology, 9, 55-72.

Golinkoff, R. M., \& Hirsh-Pasek, K. 1999. How babies talk: The magic and mystery of language development in the first three years of life. New York: Penguin, Dutton.

Hirsh-Pasek, K., Golinkoff, R. M., \& Hollich, G. 2000. An emergentist coalition model for word learning: Mapping words to objects is a product of the interation of multiple cues. In R. M. Golinkoff, K. Hirsh-Pasek, L. Bloom, L. B. Smith, A. L. Woodard, N. Akhtar, M. Tomasello, \& G. Hollich (Eds.), Becoming a word learner: A debate of lexical acquisition (pp.179-186). New York: Oxford University Press.

Hollich, G. J., Hirsh-Pasek, K., \& Golinkoff, R. M. 2000. Breaking the language barrier: An emergentist coalition model for the origins of word learning. Monographs of the Society for Research in Child Development, 65 (3, Serial No.262).

Kobayashi, H. \& Yasuda, T. 2007. A flow-ofconversation plays a role: Examining lineof-regard of young children and adults in a discrepant labeling situation. Proceedings of the Second European Cognitive Science Conference, 107-112.

Moll, H. \& Tomasello, M. 2004. 12- and 18-month-old infants follow gaze to spaces behind barriers. Developmental Science, 7, F1-F9.

Ohyabu, Y. 2004. Joint Attention - Process of Development from Newborn to Two Year and Six Months [Kyoudou chuui Shinseiji kara nisai rokkagetsu made no hattatsu katei], Tokyo: Kawashima. (in Japanese.)

Scaife, M., \& Bruner, J. 1975. "The capacity for joint visual attention in the infant". Nature, 253, 265-266.

Tomasello, M. 2001. Perceiving intentions and learning words in the second year of life. Perceiving intentions and learning words in the second year of life. In Bowerman M. \& Levinson S. (Eds.), Language Acquisition and Conceptual Development. Cambridge University Press.

Tomasello, M. \& Akhtar, N. 1995. Two-yearolds use pragmatic cues to differentiate reference to objects and actions. Cognitive Development, 10, 201-224.

Tomasello, M. \& Barton, M. 1994. Learning words in non-ostensive contexts. Developmental Psychology, 30, 639-650.

Tomasello, M., \& Farrar, J. 1986. Object permanence and relational words: A lexical training study. Journal of Child Language, 13, 495-506. 
KOBAYASHI : The Basis in Ontogeny of Language Ability

Tomasello, M. \& Haberl, K. 2003. Understanding attention: 12- and 18-month-olds know what's new for other persons. Developmental Psychology, 39, 906-912.

Yasuda, T. \& Kobayashi, H. 2008. Timing of adults' utterances and interpretation of word meanings in a discrepant labeling situation. Proceedings of the 30th Annual Meeting of the Cognitive Science Society,
675-680.

Yasuda, T. \& Kobayashi, H. 2010. KANSEI to an interval in guessing word meanings: examination of discrepant labeling situation. Journal of Japan Society of Kansei Engineering, 9(2), 377-385. (in Japanese.)

(2010. 5. 10 受稿, 2010. 5.28 受理) 